Meta

Journal des traducteurs

Translators' Journal

\title{
El desarrollo de la traduccion como disciplina en paises del Cono Sur
}

\section{Emilio Lopez et Carolina Valdivieso}

Volume 35, numéro 3, septembre 1990

La traduction dans le monde hispanolusophone

URI : https://id.erudit.org/iderudit/003516ar

DOI : https://doi.org/10.7202/003516ar

Aller au sommaire du numéro

Éditeur(s)

Les Presses de l'Université de Montréal

ISSN

0026-0452 (imprimé)

1492-1421 (numérique)

Découvrir la revue

Citer cet article

Lopez, E. \& Valdivieso, C. (1990). El desarrollo de la traduccion como disciplina en paises del Cono Sur. Meta, 35(3), 586-589. https://doi.org/10.7202/003516ar d'utilisation que vous pouvez consulter en ligne. 


\title{
EL DESARROLLO DE LA TRADUCCION COMO DISCIPLINA EN PAISES DEL CONO SUR
}

\author{
EMILIO LóPEZ Y CAROLINA VALDIVIESO \\ Pontificia Universidad Católica de Chile, Chile
}

La disciplina de traducción ha experimentado un gran avance en distintos países, debido al estudio sistemático de la actividad traductora, lo que ha permitido conformar un cuerpo de conocimentos teóricos y prácticos sobre el tema.

En el presente artículo se exponen algunas consideraciones sobre el desarrollo de la disciplina en este sector de Latinoamerica, examinando el tipo de factores que inciden en su avance. Para esto, se tomó como base uno de los aspectos tratados en una investigación orientada a conocer el estado de la formación y de la investigación en traducción en universidades del Cono Sur de América Latina, realizada por docentes de la P. Universidad Católica de Chile ${ }^{1}$. En ella se consultó a docentes y directores de 16 centros de Educación Superior de Argentina, Perú y Chile, que imparten la carrera de traducción, cuyos resultados pueden ser de interés para los demás países hispanolusohablantes.

\section{MODELO DE ANALISIS DEL DESARROLLO DE LA DISCIPLINA}

En dicho trabajo se partió del supuesto de que los programas universitarios de Traducción son los que, en mayor medida, deben constribuir al desarrollo de la disciplina a través de la formación de traductores y de la investigación en el área.

Para analizar el desarrollo de la disciplina en los centros universitarios consultados, este se abordó desde dos perspectivas:

1. una perspectiva externa, que dice relación con las características institucionales de los centros formadores de traductores, las que reflejan el nivel o estado de la traducción y, a la vez, condicionan el desarrollo de la docencia y la investigación y

2. una perspectiva interna, relacionada con la concepción subyacente de la actividad traductora en estos centros y que orienta necesariamente el tipo de formación entregada y el tipo de investigación que se haga en el área.

Desde la perspectiva externa, se consideraron características de tipo institucional tales como:

1. Orientación de la carrera : la carrera puede orientarse a la formación de profesionales, preparando cabalmente a los traductores para su desempeño laboral, y puede tener un carácter académico, que contribuye a cimentar la disciplina, tomando la traducción como objeto de estudio y reflexión.

2. Dependencia del programa : este factor, por una parte, incide el énfasis que se otorga a distintos aspectos de la formación y, por otra, es un índice del estatus de la carrera en la comunidad universitaria.

3. Perfil del cuerpo docente : las características generales del cuerpo docente (en términos de su capacitación y dedicación) deben ser concordantes con la orientación de la 
carrera para ortegar una adecuada formación al alumno y señala el potencial de desarrollo de la disciplina al interior de cada centro de formación superior.

4. Estructura curricular de la carrera: también refleja la orientación de la misma y guarda relación con la formación entregada a los traductores.

5. Estado de la investigación : este aspecto se observa a través de la cantidad, sistematización y publicación de investigaciones llevadas a cabo en cada centro, siendo un índice de la importancia que a este punto se otorga para el desarrollo de la disciplina, desde la perspectiva académica.

6. Prestigio de la carrera y de la profesión: constituye un indicador externo de la importancia que la comunidad brinda a la actividad de traducción. Este aspecto también incide en las expectativas de los alumnos y en las facilidades que el medio brinde al desarrollo de la disciplina, señalando el campo que se han abierto los traductores en el mercado ocupacional.

Desde la perspectiva interna, se consideraron las siguentes características:

1. Concepción de la disciplina : la traducción puede ser abordada desde una orientación netamente profesional, considerándosela una técnica. Sin embargo, su estudio puede llevarse a cabo desde un punto de vista científico, tecnológico o artístico. La concepción que se tenga de la disciplina condiciona tanto la formación como la investigación en la especialidad.

2. Desarrollo de la disciplina: la traducción es un fenómeno complejo que puede constituir un objeto general de estudio para distintas disciplinas, a la vez que puede ser abordada como un objeto en si misma. En este sentido, si se la considera un caso especial dentro de lo que abarcan otras disciplinas, tanto su desarrollo teórico como su metodología de investigación se enmarcarán dentro de dichas disciplinas. Así, no desarollará un cuerpo teórico ni un método propio, nutriéndose del desarrollo de las ciencias que la comprenden. En cambio, si se la estudia como un objeto en sí, independientemente de su relación evidente con otras disciplinas, desarrollará una investigación propia, con modelos teóricos y métodos particulares. Este aspecto también condiciona la formación del traductor, en términos de las habilidades que en él deban desarrollarse y orienta necesariamente la investigación en el área.

\section{SITUACION DE LA DISCIPLINA EN LOS PAISES ESTUDIADOS}

Desde la perspectiva externa, como un primer aspecto, se constató que la formación entregada por la carrera de traducción en los tres países de la muestra (Argentina, Perú y Chile) es netamente de tipo profesional. Ahora bien, si se parte del postulado que la disciplina tiene que desarrollarse en sí misma y de manera científica, nos enfrentamos a que por apuntar a una formación profesional y no a una formación académica, los objetivos de las carreras en los tres países estarían frenando el avance de la disciplina propiamente tal.

En concordancia con lo anterior, se observa que la orientación de la carrera está dirigida a satisfacer la demanda de traductores por parte del mercado laboral, con estructuras curriculares que enfatizan el aspecto fundamentalmente práctico, en desmedro de la formación teórica.

Similarmente, los docentes son traductores profesionales sin postgrado en el área, lo que resulta adecuado para dicha formación profesional, pero posterga la formación académica de la carrera. 
En los tres países estudiados, la carrera de traducción es relativamente joven lo que puede ser razón de lo anterior; sin embargo, si se desea desarrollar la traducción como disciplina, no se puede seguir postergando el establecimiento de una estructura académica.

A pesar de que en los tres países, las universidades consultadas privilegian la formación profesional de los traductores, se observó que existen diferencias en cuanto al volumen de investigación formal realizado en ellas, con la consiguiente influencia en el desarrollo de la disciplina.

En Chile, se hace más investigación formal, lo que puede deberse fundamentalmente a los siguientes factores detectados en la investigación.

En primer lugar, el tipo de contrato de los profesores, ya que en este país se detectan más jornadas completas, en tanto que en Argentina existe una proporción significativa de profesores-hora, que dedican su tiempo fundamentalmente a la docencia. En segundo lugar, la cantidad de recursos destinados a la investigación y, sobre todo, la manera de distribuirlos que incide en un mayor acceso a ellos. En particular, en Chile, existe una estructura apropiada para financiar e incentivar la investigación a diferencia de los otros dos países. Sin embargo, en Argentina y Perú, de alguna manera, los docentes realizan investigaciones, buscando por iniciativa personal diferentes fórmulas de apoyo financiero para llevar a cabo sus estudios.

Finalmente, este factor estructural guarda una estrecha relación con la importancia asignada en cada país a la actividad de traducción lo que puede condicionar indirectamente el desarrollo de la disciplina. Resulta interesante destacar que, habiendo más actividad editorial y más traducciones en Argentina, no siempre estas actividades sean llevadas a cabo por traductores profesionales, lo que ha sido señalado por los propios Directores y docentes de ese país, quienes están conscientes de la necesidad de mejorar esa situación. En Chile y Perú, donde no hay una actividad editorial similar, también ocurre que una parte de la actividad profesional es absorbida por autodidactas. Sin embargo, en Chile, los traductores profesionales han tenido una mejor aceptación por parte del mercado laboral, lo que ha suplido la falta de protección legal que es considerada una necesidad en los tres países por igual.

Frente a lo expresado más arriba, se puede considerar que las variables externas que condicionan el avance de la traducción en los países de la muestra, están relacionadas, en primer lugar, con la orientación de la carrera. Debe tratar de conseguirse, mediante estrategias viables en cada país, que las carreras adopten una orientación académica. En segundo lugar, resulta importante que las autoridades académicas brinden la posibilidad de que los docentes sigan postgrados en la especialidad y que el cuerpo docente pueda, en lo posible, tener una mayor dedicación en sus contratos. Otra variable que favorece el desarrollo de la disciplina es la cantidad de recursos para investigar con que cuenta cada escuela, por lo cual los responsables de las diferentes carreras pueden buscar formas para acceder a fondos que fomenten la actividad de investigación. La última variable en este factor guarda relación con las estrategias que se puedan diseñar para elevar el estatus profesional tanto en el aspecto gremial, como en el editorial y en el de la formación profesional de traductores a nivel superior.

Desde la perspectiva interna, se detectó un factor de tipo netamente teórico, y que dice relación con la concepción de la traducción que se tiene en cada país. Esta concepción ha orientado en especial la formación del traductor en los diversos centros universitarios, los cuales no difieren fundamentalmente entre sí al considerarlos por país, pero si se hace una comparación entre los tres países, se advertien diferencias importantes.

Se observó que en Argentina interesa en forma fundamental el dominio del idioma extranjero y el de su sintaxis. En este país, se considera que la traducción es un fenómeno linguístico cuyo proceso, desde el punto de vista teórico, no interesa tanto como la práctica 
misma de la actividad: la traducción se aprende praticando. Los traductores buscan la fidelidad al texto original y las dificuldades que surgen en la traducción provienen principalmente del conocimiento que el traductor tenga del idioma extranjero. En Argentina, la traducción es considerada una técnica y un arte, que se aprende haciendo y no teorizando. La teoría se hace en lo que aporta el estudio linguístico del texto comparado. En los centros universitarios argentinos no se busca investigar desde una perspectiva científica, sino, práctica.

En Perú, se considera que la traducción es un fenómeno comunicacional que tiene un impacto social. En este país, se estima que se debe conocer la cultura hacia la que se traduce, es decir, que el traductor debe tener un excelente dominio de su lengua materna y de su cultura para poder expresar el mensaje del texto original en la forma más cercana al lector. Los traductores no necesitan conocer la teoría. Ven la traducción no como un proceso particular que requiere una didáctica propia, sino una pedagogía que puede ser común al idioma extranjero y al castellano. En los centros universitarios peruanos no se busca un objeto propio de la traducción, sino el estudio científico de fenómenos relacionados. La investigación interesa al académico y no al traductor profesional.

En Chile interesa principalmente el proceso traductor. En este país, se considera que la traducción es un proceso sui generis que requiere una didáctica particular y apropiada. La habilidad traductora suple, en cierta medida, el desconocimiento de las lenguas. No basta saber idiomas extranjeros para ser capaz de traducir. La traducción no es completamente abarcada por la linguística, sino que es una habilidad distinta a ella. El traductor debe conocer la teoría para aplicarla a la práctica. Como ciencia puede abocarse al estudio de este proceso. En los centros universitarios chilenos se tiende a buscar el objeto propio de la ciencia de la traducción y sus métodos de investigación.

Frente a la diferente concepción de la traducción que existe en cada uno de los países, resulta evidente que el desarrollo de la investigación en cada uno de ellos va a estar relacionado con los modelos teóricos que subyacen en cada una de estas concepciones. Lo importante, por un lado, es que cada escuela tenga conciencia de dichos modelos para tratar de lograr un adecuado ajuste entre la formación teórica y la formación práctica que se le da a los futuros traductores y, por otro, es que en cada programa de traducción se diseñe un plan de desarrollo de la investigación acorde con las orientaciones y las necesidades específicas de cada escuela.

Es indudable que, por varias razones, el desarrollo del estudio de la traducción, al igual que el de cualquier otra disciplina, contribuye al desarrollo científico y tecnológico en general, cuyo análisis excede la finalidad de este artículo. Entre ellas, nos parecen de especial relevancia la pérdida de una identidad cultural y la creciente dependencia que con respecto a los países industrializados tienen nuestros países por el hecho de ser sólo receptores de la investigación realizada en los centros universitarios extranjeros, lo que no siempre coincide con los problemas presentes en nuestra realidad.

\section{NOTES}

1. «Diagnóstico acerca del estado de la formación y de la investigación en el campo de la traducción en universidades chilenas, argentinas y peruanas», Proyecto $\mathrm{n}^{0} 57 / 88$, financiado por la Dirección de Investigación de la P. Universidad Católica de Chile, realizado en 1988, por ILeana Cabrera, Soledad Dargham, Jaime Hagel, Emilio Lopez y Carolina Valdivieso. 